\title{
Hyperinsulinaemic hypoglycaemia, renal Fanconi syndrome and liver disease due to a mutation in the HNF4A gene
}

\author{
María Clemente', Alejandro Vargas'ㄹ, Gema Ariceta², Rosa Martínez³, \\ Ariadna Campos' ${ }^{1}$ and Diego Yeste' ${ }^{1}$ \\ 1Paediatric Endocrinology Unit, Department of Paediatrics and 2Paediatric Nephrology Service, \\ Vall d'Hebron Hospital, Autonomous University of Barcelona, CIBERER, Barcelona, Spain, and \\ ${ }^{3}$ Hospital Universitario Cruces, UPV-EHU, BioCruces, CIBERDEM, CIBERER, Bilbao, Spain
}

\author{
Correspondence \\ should be addressed \\ to $\mathrm{M}$ Clemente \\ Email \\ mclement@vhebron.net
}

\section{Summary}

HNF4A gene mutations have been reported in cases of transient and persistent hyperinsulinaemic hypoglycaemia of infancy $(\mathrm{HHI})$, particularly in families with adulthood diabetes. The case of a patient with HHI, liver impairment and renal tubulopathy due to a mutation in HNF4A is reported.

\section{Learning points:}

- Urine specimen study in cases of $\mathrm{HHI}$ with diazoxide response is necessary to rule out specific metabolic conditions (L-3-hydroxyacyl-coenzyme A dehydrogenase deficiency) or tubular renal involvement.

- Hyperinsulinaemic hypoglycaemia due to the heterozygous mutation (p.Arg63Trp, c. 187C > T) in the HNF4A gene is associated with renal tubulopathy and liver involvement.

- Follow-up of patients diagnosed of $\mathrm{HHI}$ is mandatory to detect associated conditions.

\section{Background}

Hyperinsulinaemic hypoglycaemia of infancy (HHI) is the most frequent cause of persistent neonatal hypoglycaemia and hypoglycaemia in infancy and is caused by dysregulation in insulin secretion. Molecular bases are known in $50-60 \%$ of patients. To date, eleven genes have been implicated, and the most frequent cause is mutations in the genes encoding K-ATP channel subunits. Heterozygous mutations in the HNF4A gene have been reported in cases of transient and persistent $\mathrm{HHI}$, particularly in families with neonatal hypoglycaemia and adulthood diabetes (1). The HNF4A gene encodes for the transcription factor hepatocyte nuclear 4a (HNF4a) that controls the expression of genes involved in glucosestimulated insulin secretion; its mutations were first known to be the cause of monogenic diabetes type 1 (MODY 1).
Herein, we present a case of hyperinsulinaemic hypoglycaemia with a mutation in HNF4A and renal tubulopathy; this characteristic phenotype has been recently described and very few cases have been reported worldwide.

\section{Case presentation}

A full-term large-for-gestational age boy (birth weight $4250 \mathrm{~g}+2.7$ S.D. and length $55 \mathrm{~cm}+3.29$ s.D., head circumference $37 \mathrm{~cm}+1.53$ s.D.) developed hypoglycaemia on the first day of life. He was the second child of nonconsanguineous Caucasian parents. High carbohydrate i.v. administration was required to maintain euglycaemia (up to $17 \mathrm{mg} / \mathrm{kg} / \mathrm{min}$ ). Laboratory results showed 
inappropriately low betahydroxybutyrate plasma levels (10.2 $\mu \mathrm{mol} / \mathrm{L})$, inappropriate insulin (6.1 IU/L) and C-peptide levels $(2.7 \mathrm{ng} / \mathrm{mL})$ and low cortisol levels $(1.3 \mu \mathrm{g} / \mathrm{dL}$ and $5.2 \mu \mathrm{g} / \mathrm{dL})$ in hypoglycaemia. The diagnosis of congenital hyperinsulinaemic hypoglycaemia was established. However, owing to low cortisol levels, hydrocortisone was started at $10 \mathrm{mg} / \mathrm{m}^{2} /$ day without response. Function of other hypophyseal hormones was normal (thyroid hormones and testosterone levels were normal). Glucose in urine strips was detected on more than one occasion. Diazoxide was started at a dose of $8 \mathrm{mg} / \mathrm{kg} /$ day $24 \mathrm{~h}$ after initiation of hydrocortisone and intravenous glucose could be stopped, normalising total carbohydrate requirements. As the patient did not present hypoglycaemia after $6 \mathrm{~h}$ of fasting, he was discharged at 6 weeks of age on diazoxide at a dose of $6 \mathrm{mg} / \mathrm{kg} /$ day and with a nasogastric tube owing to feeding difficulties. Shortly after discharge, the diazoxide dose needed to be reduced owing to hyperglycaemia and was stopped at the age of three months since the glycaemia reached $300 \mathrm{mg} / \mathrm{dL}$ even with a dose lower than $3 \mathrm{mg} / \mathrm{kg} /$ day. Treatment was based only on frequent feeds, both oral and by nasogastric tube that was maintained until the age of 18 months.

At six months of age, the patient had failure to thrive and mild hepatomegaly ( $3 \mathrm{~cm}$ below the costal margin). Laboratory tests showed high serum transaminase levels (AST 80-90IU/L, ALT 40-50IU/L and GGT 60-100 IU/L). Laboratory tests for hepatotropic viruses and autoimmunity were negative. Liver biopsy was not performed as rises in liver enzymes were transient.

During follow-up, a progressive increase was observed in alkaline phosphatase (800-1384IU/L), together with vitamin D deficiency $(25 \mathrm{OHD}<20 \mathrm{ng} / \mathrm{mL}$ ) with low serum phosphate (3-3.2 mg/dL) but normal calcium $(9.7 \mathrm{mg} /$ $\mathrm{dL})$ and magnesium $(2.48 \mathrm{mg} / \mathrm{dL})$ levels. Treatment with cholecalciferol was prescribed; however, the child gradually developed rickets with craniotabes, wrist widening, genu varum and motor impairment; findings on knee X-ray at 12 months of age were typical of rickets (Fig. 1). Further complementary tests at 18 months of age revealed nongap metabolic acidosis ( $\mathrm{pH} 7.27$, bicarbonate 19.3) with acidified urine ( $\mathrm{pH}$ 5.5) suggestive of proximal tubular acidosis, glycosuria with generalised aminoaciduria and tubular type proteinuria ( $\beta 2$ microglobulin $>4 \mathrm{mg} / \mathrm{L}$ ), in addition to hypophosphatemia of renal origin (serum $\mathrm{P}$ $3.2 \mathrm{mg} / \mathrm{dL}$ and TRP 72\%), hypercalciuria (UCa/Cr $0.5 \mathrm{mg} /$ $\mathrm{mg}$ and UCa $3.7 \mathrm{mg} / \mathrm{kg} /$ day) and carnitine deficiency (total carnitine $29.3 \mu \mathrm{mol} / \mathrm{L})$. 25OHD levels were normal at that time $(30 \mathrm{ng} / \mathrm{mL})$, but PTH was elevated $(155 \mathrm{pg} / \mathrm{mL})$.

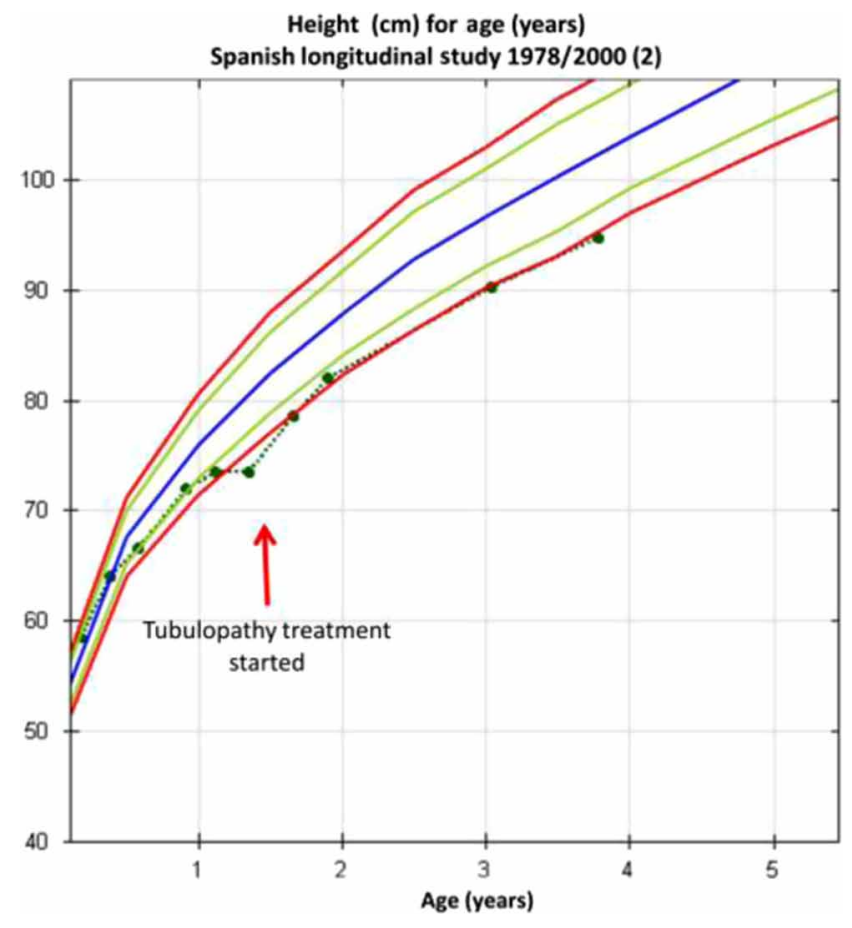

Figure 1

Growth chart over first 3 years of life.

Fanconi-type proximal complex tubulopathy was diagnosed. By that time, he had lost 5.8 standard deviations in height and 4 standard deviations in weight since birth (Fig. 2). Supplementary oral treatment with phosphate, sodium bicarbonate, carnitine and 1-alphacalcidiol was started immediately.

\section{Investigation}

Genetic testing for KCNJ11 and ABCC8 genes did not detect any mutation. Responsiveness to diazoxide and glucosuria pointed to $H N F 4 A$ study, which revealed a heterozygous mutation in exon 2 of the HNF4A gene: p.Arg63Trp,

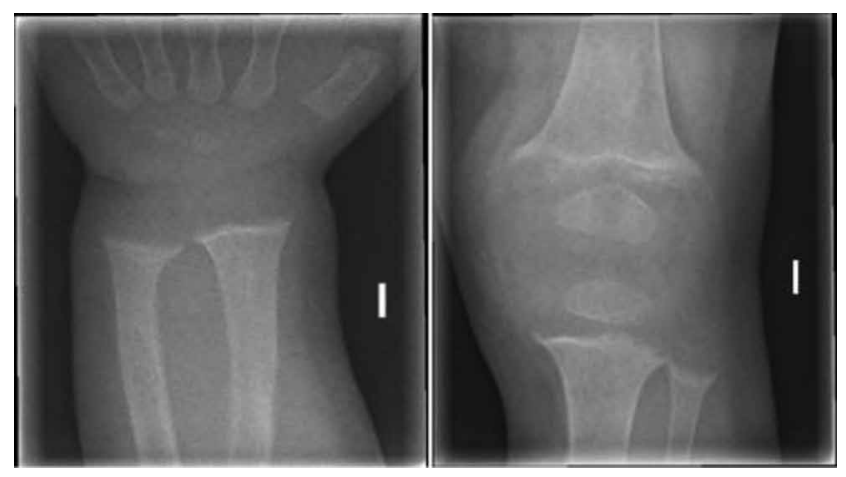

Figure 2

Wrist and knee X-rays at 16 months of age. 
c.187C $>\mathrm{T}$ (reference sequence: NM_175914.4). Both parents tested negative for the mutation.

\section{Treatment}

Diazoxide was started, and intravenous glucose could be stopped. On more than one occasion, the dose needed to be reduced owing to hyperglycaemia and had to be stopped by the age of three months. At six months of age, the patient was observed to have failure to thrive and mild hepatomegaly with slightly high serum transaminase levels. Tests for hepatotropic viruses and autoimmunity were negative. During follow-up, a progressive increase in alkaline phosphatase (800-1384 IU/L), vitamin D deficiency (25OHD: $<20 \mathrm{ng} / \mathrm{mL}$ ), low serum phosphate (3-3.2 mg/dL), but normal calcium and magnesium levels were observed. The child gradually developed rickets despite treatment with cholecalciferol. Further complementary tests revealed proximal tubular acidosis, glycosuria with generalised aminoaciduria and tubular type proteinuria, in addition to hypophosphataemia, hypercalciuria and carnitine deficiency. Fanconi-type proximal complex tubulopathy was diagnosed, and supplementary oral treatment was initiated. After treatment was started, the patient gained weight and height and recovered from his skeletal abnormalities.

\section{Outcome and follow-up}

At 3 years and 9 months of age, the patient has currently no signs of active rickets and, despite persistent Fanconi syndrome, his metabolic control is satisfactory. Cognitive and motor development are age adequate; weight (14.3 kg, -1.4 s.D.) and height ( $95 \mathrm{~cm},-2$ s.D.) have normalised with growth catch-up (2). He is now able to fast for eight hours; however, he remains on a 02:00 h intake that is being tapered. Minimal hepatomegaly and mildly increased transaminases (AST 98IU/L, ALT $63 \mathrm{IU} / \mathrm{L}$ at the last control) remain, with transient peaks during episodes of viral upper respiratory tract infection or gastroenteritis.

\section{Discussion}

The association between HHI and HNF4A mutations has been found in $0.5-2.4 \%$ of patients and was first reported by Pearson et al. (3). That study also reported a link between those mutations and macrosomia in a significant percentage of carriers. Nevertheless, it was not until 2010 that the mutation in our patient (p.Arg63Trp, c.187C > T also reported as p.Arg76Trp (p.R76W), c.226C>T) was found to cause $\mathrm{HHI}$, although the only patient reported to have it was not phenotypically described (4). A second case with the same mutation was reported in 2012. That patient had the same features as our patient, including the liver disorder, together with a liver biopsy, which showed abundant cytoplasmic glycogen with mild portal inflammation and fibrosis (5). In 2014, six more cases with our patient's mutation were reported, including the first previously published in 2010 and without a phenotypical description. These six patients had no liver disorder and, unlike the patient we report, also had 'atypical' Fanconi syndrome with hypercalciuria with relative hypocalcaemia, hypermagnesaemia, nephrocalcinosis and renal impairment. Two patients had developed diabetes mellitus at 12 and 20 years of age respectively (6). Recently, Numakura et al. (7) and Improda et al. (8) each reported two additional cases, one of which had transient liver disease; the case described by Numukara presented hepatomegaly and raised serum transaminases at five months of age with normalisation at the age of three and that described by Improda had prolonged conjugated hyperbilirubinaemia which normalised over the first 3 months of life. Those authors suggested that transient liver enlargement could be a mutation-specific characteristic of p.R76W, taking into consideration that liver-specific $H N F 4 A$-knockout mice had hepatomegaly and that HNF4A regulates the expression of $S L C 2 A 2$, a mutation which is a cause of Fanconi-Bickel syndrome $(5,7)$. However, not all the described patients with the R76W mutation presented liver impairment. Liver disease in our case has been permanent to date.

HNF- $4 \alpha$ is known to regulate selected genes involved in insulin secretion; however, the precise molecular mechanisms by which $H N F 4 A$ mutations cause insulin hypersecretion are not clearly understood (1). There appears to be a relationship with PPARa levels. PPARa is a transcription factor that regulates fatty acid $\beta$-oxidation; lower levels of this factor might reduce $\beta$-oxidation of fatty acids, thereby increasing the availability of lipid signalling molecules that are necessary for insulin exocytosis. This would lead to abnormal insulin secretion during fasting (9).

Our case provides further evidence of the role of the $H N F 4 A$ gene in the renal proximal tubule (PT). Potential pathogenic mechanisms accounting for the generalised PT dysfunction and thus the occurrence of Fanconi syndrome in patients with $H N F 4 A$ mutations are unknown; however, the mutation-specific effect and involvement of major regulatory genes have been postulated (6). The $H N F 4 A$ 
R76W mutation causes an atypical dominant Fanconi syndrome in addition to the beta cell phenotype. Other inherited diseases causing Fanconi syndrome and neonatalonset hypoglycaemia, such as galactosaemia, tyrosinemia or mitochondrial diseases, were ruled out in our patient since his hypoglycaemia was due to hyperinsulinism. Further, Bickel-Fanconi syndrome is another Fanconi syndrome of hereditary origin with hypoglycaemia and liver impairment; however, symptoms usually appear later in infancy and hypoglycaemia is due to impaired liver release of glucose due to a GLUT2 deficiency. These facts also facilitated the differential diagnosis in our case.

The low cortisol levels found at diagnosis in our patient were noteworthy. This feature is relatively common among HHI patients and appears to be related to a lack of drive from the hypothalamic-pituitary axis, with inappropriately low plasma adrenocorticotropic hormone concentrations at the time of hypoglycaemia (10). The mechanism of this low response is not clear; the presence of K-ATP channels in the ventromedial hypothalamus (involved in glucose sensing) suggests that these channels might be closed in HHI, thereby impairing the counter-regulatory response to hypoglycaemia. However, this blunted counter-regulatory response has also been described in patients with no mutations in $A B B C 8 / K C N J 11$ genes. Hypoglycaemia-associated autonomic failure (HAAF) due to repeated hypoglycaemia has also been postulated. Further studies will be required to elucidate the counter-regulatory hormonal responses in patients with $\mathrm{CHI}$.

The long-term follow-up of our patient should include glycaemic control in the search for the possible future onset of diabetes mellitus.

Our case adds to the understanding and subtleties of this rare and recently described mutation uniquely causing HHI and Fanconi syndrome. Our patient has permanent liver involvement and no atypical features in his FS, unlike the cases reported by Hamilton et al. (6).

It is clear that subtle initial laboratory results in our patient might have yielded clues and an earlier diagnosis could have been made. We hope this report will help to raise awareness of the possibility of FS and liver disorder when faced with children with HHI.

\section{Patient's perspective}

Being parents, the most difficult thing for us was the uncertainty. It was at the Neonatal Care Unit where we were told about his first diagnosis: congenital hyperinsulinism. Our medical team told us that the causes and prognosis could be multiple. After a 6-week period at the Neonatal Unit, they let us take him home. Our endocrinologist, who has been accompanying us all these years, told us: 'please be patient'. Sometimes it has been difficult for us to keep calm, especially when he was 18 months old. We had the feeling that, beyond his first diagnosis, more and more new conditions were affecting him every now and then as he was growing up, such as proximal tubulopathy and raised transaminase levels. We could only 'relax' when the results of the genetic test arrived. The mutation of the gene HNFA4 was finally providing a reason for the whole clinical picture. Our son is now leading a normal life thanks to his regular feeding. The most difficult thing for us is to make him take his medicines, but as he was born with all these disorders, he is fully aware of the benefits of doing so, despite being just a 4-year-old child.

\section{Declaration of interest}

The authors declare that there is no conflict of interest that could be perceived as prejudicing the impartiality of the research reported.

\section{Funding}

This research did not receive any specific grant from any funding agency in the public, commercial or not-for-profit sector.

\section{Patient consent}

Written informed consent was obtained from the patient's guardian for publication of the submitted article and accompanying images.

\section{References}

1 Nessa A, Rahman SA \& Hussain K 2016 Hyperinsulinemic hypoglycemia - the molecular mechanisms. Frontiers in Endocrinology 7 29. (doi:10.3389/fendo.2016.00029)

2 Carrascosa Lezcano A, Fernández García JM, Fernández Ramos C, Ferrández Longás A, López-Siguero JP, Sánchez González E, Sobradillo Ruiz B \& Yeste Fernández D 2008 Grupo Colaborador Español. Spanish cross-sectional growth study 2008. Part II. Height, weight and body mass index values from birth to adulthood. Anales de Pediatría 68 552-569. (doi:10.1016/ j.anpedi.2010.03.017)

3 Pearson ER, Boj SF, Steele AM, Barrett T, Stals K, Shield JP, Ellard S, Ferrer J \& Hattersley AT 2008 Macrosomia and hyperinsulinaemic hypoglycaemia in patients with heterozygous mutations in the HNF4A gene. PLoS Medicine 4 e118. (doi:10.1371/journal. pmed.0040118)

4 Flanagan SE, Kapoor RR, Mali G, Cody D, Murphy N, Schwahn B, Siahanidou T, Banerjee I, Akcay T, Rubio-Cabezas O, et al. 2010 Diazoxide-responsive hyperinsulinemic hypoglycemia caused by HNF4A gene mutations. European Journal of Endocrinology 162 987-992. (doi:10.1530/EJE-09-0861)

5 Stanescu DE, Hughes N, Kaplan B, Stanley CA \& De León DD 2012 Novel presentations of congenital hyperinsulinism due to mutations in the MODY genes: HNF1A and HNF4A. Journal of Clinical Endocrinology and Metabolism 97 E2026-E2030. (doi:10.1210/jc.2012-1356) 
6 Hamilton AJ, Bingham C, McDonald TJ, Cook PR, Caswell RC, Weedon MN, Oram RA, Shields BM, Shepherd M, Inward CD, et al. 2014 The HNF4A R76W mutation causes atypical dominant Fanconi syndrome in addition to a $\beta$ cell phenotype. Journal of Medical Genetics 51 165-169. (doi:10.1136/jmedgenet-2013-102066)

7 Numakura C, Hashimoto Y, Daitsu T, Hayasaka K, Mitsui T \& Yorifuji T 2015 Two patients with HNF4A-related congenital hyperinsulinism and renal tubular dysfunction: a clinical variation which includes transient hepatic dysfunction. Diabetes Research and Clinical Practice 10 e53-e55. (doi:10.1016/j.diabres.2015.03.005)

8 Improda N, Shah P, Güemes M, Gilbert C, Morgan K, Sebire N, Bockenhauer D \& Hussain K 2016 Hepatocyte nuclear factor-4 alfa mutation associated with hyperinsulinaemic hypoglycaemia and atypical renal Fanconi syndrome: expanding the clinical phenotype. Hormone Research in Paediatrics 86 337-341. (doi:10.1159/000446396)

9 Gremlich S, Nolan C, Roduit R, Burcelin R, Peyot ML, DelghingaroAugusto V, Desvergne B, Michalik L, Prentki M \& Wahli W 2005 Pancreatic islet adaptation to fasting is dependent on peroxisome proliferator-activated receptor alfa transcriptional up-regulation of fatty acid oxidation. Endocrinology 146 375-382. (doi:10.1210/ en.2004-0667)

10 Hussain K, Hindmarsh P \& Aynsley-Green A 2003 Neonates with symptomatic hyperinsulinemic hypoglycemia generate inappropriately low serum cortisol counterregulatory hormonal responses. Journal of Clinical Endocrinology and Metabolism $\mathbf{8 8}$ 4342-4347. (doi:10.1210/jc.2003-030135)

Received in final form 17 November 2016 Accepted 14 February 2017 